\title{
Effect of Subconjunctivally Injected, Liposome-Bound, Low-Molecular-Weight Heparin on the Absorption Rate of Subconjunctival Hemorrhage in Rabbits
}

\author{
Jun-Woong Moon, ${ }^{1,2}$ Yun-Kyoung Song, ${ }^{2,3,4}$ Jun-Pil Jee, ${ }^{3}$ Cbong-Kook Kim, ${ }^{3}$ \\ Ho-Kyung Cboung, ${ }^{5}$ and Jeong-Min Hwang ${ }^{1,6}$
}

Purpose. To investigate the effect of subconjunctival injection of liposome-bound, low-molecular-weight heparin (LMWH) on the absorption rate of subconjunctival hemorrhages.

MethoDs. Subconjunctival hemorrhages were induced in both eyes of 30 rabbits by the subconjunctival injection of $0.1 \mathrm{~mL}$ of autologous blood from auricular marginal veins. After 8 hours, randomized subconjunctival injections of one of three materials were made: $5 \mathrm{IU} / \mathrm{mL}$ liposome-bound $\mathrm{LMWH}(0.1 \mathrm{~mL})$ in 18 eyes (group A), only liposomes $(0.1 \mathrm{~mL}$ ) in 14 eyes (group B), the free form of LMWH ( $5 \mathrm{IU} / \mathrm{mL}, 0.1 \mathrm{~mL}$ ) in 14 eyes (group C), or no injection in 14 eyes (group D). Subconjunctival hemorrhages were photographed with a digital camera at 8, 24, 48, 72,96 , and 120 hours after induction of subconjunctival hemorrhages, sized with an image analyzer, and compared between groups.

RESults. Subconjunctival hemorrhages were absorbed faster in group A (liposome-bound LMWH injected) than in with group B (liposome injected). Comparison of groups A and C (free LMWH injected) showed statistical differences in the absorption rates at 96 and 120 hours except at 24, 48, and 72 hours. The mean elapsed time for the complete resorption of subconjunctival hemorrhages was shortest in group A among four groups, whereas group B and the control showed no significant differences. The ocular and systemic absorption of LMWH were significantly lower after injection of the liposome-bound than the free form.

Conclusions. The subconjunctival injection of liposome-bound LMWH appears to enhance subconjunctival hemorrhage absorption in rabbits. (Invest Ophthalmol Vis Sci. 2006;47: 3968-3974) DOI:10.1167/iovs.05-1345

From the ${ }^{1}$ Department of Ophthalmology, Seoul National University College of Medicine, Artificial Eye Center of Clinical Research Institute, Seoul National University Hospital, Seoul, Korea; ${ }^{3}$ Laboratory of Excellency for Drug and Gene Delivery, College of Pharmacy, Seoul National University, Seoul, Korea; ${ }^{4}$ Test and Analytical Laboratory, Korea Food and Drug Administration, Seoul, Korea; the ${ }^{5}$ Department of Ophthalmology, Seoul National University Boramae Hospital, Seoul, Korea; and the ${ }^{6}$ Department of Ophthalmology, Seoul National University Bundang Hospital, Seongnam, Korea.

${ }^{2}$ Contributed equally to the work and therefore should be considered equivalent authors.

Submitted for publication October 13, 2005; revised March 11 and April 24, 2006; accepted July 15, 2006

Disclosure: J.-W. Moon, None; Y.-K. Song, None; J.-P. Jee, None; C.-K. Kim, None; H.-K. Choung, None; J.-M. Hwang, None

The publication costs of this article were defrayed in part by page charge payment. This article must therefore be marked "advertisement" in accordance with 18 U.S.C. $\$ 1734$ solely to indicate this fact.

Corresponding author: Chong-Kook Kim, Laboratory of Excellency for Drug and Gene Delivery, College of Pharmacy, Seoul National University, San 56-1, Shillim-Dong, Kwanak-Gu, Seoul 151-742, Korea; ckkim@plaza.snu.ac.kr. ubconjunctival hemorrhage is exposed to view and can be $\checkmark$ troublesome from a cosmetic perspective. However, few therapeutic trials have been attempted to enhance its absorption, ${ }^{1}$ and ophthalmologists tend to disappoint patients by advising that they wait until absorption occurs spontaneously.

Low molecular weight heparin (LMWH) produced from chemical or enzymatic hydrolytic cleavage of unfractionated heparin is an antithrombotic drug with an apparent molecular weight in the range of 3 to $9 \mathrm{kDa}$. LMWH is an improved anticoagulant compared with unfractionated heparin with respect to a longer half-life, higher bioavailability, less bleeding propensity, and less binding to non-anticoagulant-related plasma proteins and platelets. $^{2}$ It inhibits thrombin and thus suppresses the cascade of reactions that lead to blood clotting and the formation of a fibrin meshwork in vitro and in vivo. Thus, it could prevent clots from becoming larger and causing more serious problems in such conditions as ischemic heart disease and acute pulmonary embolism. ${ }^{3,4}$

Although it is generally believed that heparin does not dissolve blood clots that have already been formed, there have been several reports regarding the fibrinolysis-enhancing effects of heparin. ${ }^{2,5-7}$ Heparin prevents the formation of stable fibrin clots by inhibiting the activation of fibrin stabilizing factor. $^{2}$ The administration of LMWH is known to induce the release of antiplatelet substances like prostacyclin, and of fibrinolytic activators such as tissue plasminogen activator, the latter of which converts plasminogen into plasmin and activates several matrix metalloproteinases. ${ }^{5-7}$ It has been also reported that fibrinolysis may be suppressed by thrombin. ${ }^{7}$

Considering these antithrombotic and fibrinolysis-enhancing activities of heparin, it is possible that the subconjunctival application of heparin affects the absorption of subconjunctival hemorrhage. However, as heparin has a narrow therapeutic range, the subconjunctival applicability of LMWH is limited by the potential risk of systemic and ocular side effects. Liposomes may be an appropriate ocular drug delivery system to overcome these limitations, as subconjunctival liposomes may be related to the sustained release and retentive effects at the site of injection of the encapsulated drug. ${ }^{8,9}$

The present scenario is full of opportunities in this regard, as it may be that controlled-release of liposome-bound LMWH would be helpful in the absorption of subconjunctival hemorrhages. Few studies have been conducted to investigate the possibility. We investigated the effect of liposome-bound LMWH on the absorption rate of subconjunctival hemorrhages.

\section{Materials ANd Methods}

The lipids of liposomes, 1,2-dioleoyl-3-trimethylammonium-propane (chloride salt, DOTAP, >99\%) and cholesterol, were purchased from Avanti Polar Lipids Inc. (Birmingham, AL). LMWH (80 IU/mg, $\sim 7 \mathrm{kDa}$ ) was from Daihan Pharmaceutical Co. (Seoul, South Korea). Fifty-four New Zealand White rabbits, weighing 2.0 to $3.0 \mathrm{~kg}$, were used in the study. All experimental methods and animal care procedures con- 
formed to the ARVO Statement for the Use of Animals in Ophthalmic and Vision Research.

\section{Preparation and Size Measurement of Liposome-Bound LMWH}

Liposome-bound LMWH was prepared by hydration of a lipid film with a drug solution, as previously described, with slight modification in a single large batch. ${ }^{10}$ Briefly, cholesterol and DOTAP were dissolved in $2 \mathrm{~mL}$ of chloroform at a molar ratio of 1:1. The solvent was removed under reduced pressure at $50^{\circ} \mathrm{C}$ to $60^{\circ} \mathrm{C}$ in a rotary evaporator (model RE-121; Büchi Labortechnik, Essen, Germany) to leave a thin lipid film on a round-bottomed flask. Residual organic solvent was removed by flushing with nitrogen for 1 hour. Sterile saline containing LMWH (80 $\mathrm{IU} / \mathrm{mg}, \sim 7 \mathrm{kDa}$ ) at a concentration of $10 \mathrm{IU} / \mathrm{mL}$ was added to the film to create $2 \%$ (wt/vol) lipid suspension. After vigorous vortexing for 1 hour, the resultant liposomes were sonicated in a bath type sonicator (Branson Ultrasonic Co. Danbury. CT) for 1 hour at room temperature and extruded (Avestin Co., Ottawa, ON, Canada) twice through polycarbonate membranes (Nuclepore; Whatman, Clifton, NJ), once at a pore size of $1 \mu \mathrm{m}$ followed by $200 \mathrm{~nm}$, under nitrogen pressure (300 $\mathrm{kPa}$ ). After extrusion, the liposomal suspension was mixed with $0.02 \%$ of benzalkonium chloride (preservative) and stored at $4^{\circ} \mathrm{C}$. The mean size of the liposome-bound LMWH was $546 \pm 41.94 \mathrm{~nm}(n=5)$.

\section{Evaluation of In Vitro Release of LMWH from Liposomes}

The liposome-bound LMWH formulations were placed in a closed vial and immersed in a shaking water bath at $37^{\circ} \mathrm{C}$. At $24,48,72,96$, and 120 hours after preparation of liposome-bound LMWH, the in vitro release rate of LMWH from liposomes was determined by separating liposomes from the suspension medium by ultracentrifugation (Optima TLX ultracentrifuge; Beckman, Fullerton, CA) in a rotor (TLA 100.3; Beckman) at room temperature, $150,000 \mathrm{~g}$ for 90 minutes. ${ }^{11}$ Then, the supernatant was removed and replaced with the same quantity of sterile saline for subsequent analysis. LMWH content in the supernatant was determined by a modified colorimetric method (Azure II; Sigma-Aldrich, St. Louis, MO) at each interval. ${ }^{12}$ The cumulative release rate was defined as follows: $(C / T) \times 100(\%)$, where $C$ is the cumulative amount of LMWH detected only in the supernatant, and $T$ is the total amount of LMWH in liposome formulations.

\section{Induction of Subconjunctival Hemorrhages}

General anesthesia was achieved intramuscularly with 30 to $45 \mathrm{mg} / \mathrm{kg}$ of ketamine hydrochloride and 5 to $10 \mathrm{mg} / \mathrm{kg}$ of xylazine hydrochloride and topical anesthesia with proparacaine hydrochloride. Autologous blood sampled from the auricular marginal vein was subconjunctivally injected into both eyes of all rabbits $(0.1 \mathrm{~mL}, 1$ to $2 \mathrm{~mm}$ from the superior limbus) with a $1-\mathrm{mL}$ syringe and 30 -gauge needle with the bevel down. Every effort was made to avoid any vascular injury at the ocular surface.

\section{Grouping}

Eight hours after induction of subconjunctival hemorrhages, the material to be injected for each eye of 30 rabbits was assigned by block randomization: group A (18 eyes): $0.1 \mathrm{~mL}$ of liposome-bound LMWH ( 5 $\mathrm{IU} / \mathrm{mL}$ ); group B (14 eyes): $0.1 \mathrm{~mL}$ of liposomes; group C (14 eyes): 0.1 $\mathrm{mL}$ of the free form of LMWH $(5 \mathrm{IU} / \mathrm{mL})$; and group D: (14 eyes): no injection. Block randomization was performed by selecting blindly one of cards, on which were written the names of material to be injected. A preliminary experiment demonstrated no significant effects on the absorption rate of subconjunctival hemorrhages in the fellow untreated eyes after treatment of one eye with liposome-bound or free form LMWH, thus allowing use of both eyes simultaneously. The participants, who performed block randomization, subconjunctival

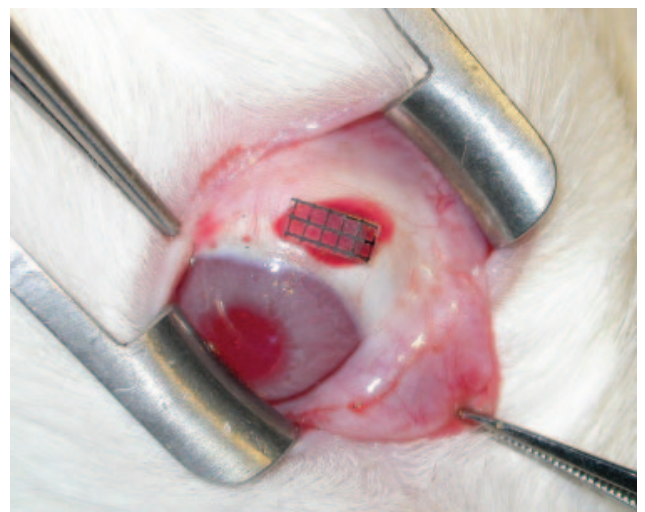

FigURE 1. Measurement of the size of a subconjunctival hemorrhage. A translucent $5 \times 2-\mathrm{mm}^{2}$ bar, marked off in $1 \mathrm{~mm}$, was placed on a subconjunctival hemorrhage. A photograph was taken with a digital camera positioned normally from the center of the lesion at a fixed distance $(21 \mathrm{~cm})$ and magnification $(\times 125)$. Lesion sizes were calculated with an image analyzer.

injections, and measurement of the sizes of subconjunctival hemorrhages, were blind to the types of the injected materials.

\section{Measurement of the Subconjunctival Hemorrhage Size}

Subconjunctival hemorrhage was photographed with a digital camera (Coolpix 950; Nikon, Tokyo, Japan) at 8, 24, 48, 72, 96, and 120 hours after the hemorrhage induction. The digital camera was positioned perpendicular to the center of the lesion at a fixed distance $(21 \mathrm{~cm})$ and magnification $(\times 125)$. A translucent bar, $5 \times 2 \mathrm{~mm}^{2}$ in size marked with $1-\mathrm{mm}$ graduations, was placed onto the central area of subconjunctival hemorrhage as a reference for sizing (Fig. 1).

A prominent subconjunctival bleb was produced by injecting autologous blood, and this enlarged and flattened after 4 to 5 hours of the induction. Therefore, the sizes of subconjunctival hemorrhages at 8 hours after induction were defined as basal levels. The digitalized images of subconjunctival hemorrhages were sized using an image analyzer (Image Pro-Plus, ver. 3.0.1; Media Cybernetics, Silver Spring, MD).

\section{Evaluation of the Absorption Rates of Subconjunctival Hemorrhage}

Two parameters were used for the evaluation of the absorption rates of subconjunctival hemorrhages. One of these parameters was the amount of decrease in the size of the subconjunctival hemorrhage from baseline size (i.e., the size of the hemorrhage at 8 hours after the induction), which were compared between groups at 24, 48, 72, 96, and 120 hours after induction of the hemorrhages. In addition, the mean elapsed times required for the complete absorption of subconjunctival hemorrhages were compared among groups.

\section{Evaluation of Ocular and Systemic Absorption and Intraocular Side Effects of LMWH}

The remaining 24 rabbits were used for the following experiments. Eight hours after induction of subconjunctival hemorrhages in one eye of each rabbit (24 eyes) as described earlier, subconjunctival injection of liposome-bound LMWH (12 rabbits) or the free form of LMWH (12 rabbits) was randomly performed. Slit lamp and fundus examinations were performed after pupil dilation with $1 \%$ tropicamide and $2.5 \%$ phenylephrine hydrochloride eye drops before and at 24, 48, 72, and 120 hours after subconjunctival hemorrhage induction with subsequent liposome-bound or free LMWH injection. The developments of corneal or lens opacity and inflammation or hemorrhage in the anterior 
chamber and vitreous were evaluated. Then, three rabbits randomly chosen from each group at 24, 48, 72, and 120 hours after subconjunctival hemorrhage induction were killed for the measurement of LMWH activity in the plasma and ocular tissues of the treated and fellow untreated eyes. After anesthesia with intramuscular injection of ketamine hydrochloride and xylazine hydrochloride, $5 \mathrm{~mL}$ of blood was sampled from a marginal auricular vein and centrifuged. The concentration of LMWH in plasma was determined by a LMWH colorimetric plasma assay with a sensitivity of 0.1 to $1 \mathrm{IU} / \mathrm{mL}$ (Coatest; Chromogenix Ins., Milano, Italy).

The rabbits were then killed with an overdose of intravenous pentobarbital sodium and the eyes enucleated. To evaluate the ocular absorption of LMWH in the treated and fellow untreated eyes, the anticoagulant activities in the various ocular tissues such as aqueous humor, vitreous, and a piece of conjunctiva and sclera adjacent to the injection site were measured. Briefly, aqueous taps $(0.1-0.2 \mathrm{~mL})$ were performed with a $1-\mathrm{mL}$ syringe with 30 -gauge needle via a limbal paracentesis site. After a circumferential full-thickness incision through the sclera and choroid was made $2.5 \mathrm{~mm}$ from the limbus, the vitreous body was harvested by expression. In addition, a piece of conjunctiva and sclera $\left(5 \times 5 \mathrm{~mm}^{2}\right)$ around the injection site were removed. The harvested tissues were kept in tubes at $-70^{\circ} \mathrm{C}$ for subsequent analysis. The amounts of LMWH in sclera, conjunctiva, and vitreous were determined by cutting the tissues into small pieces. The tissues were pooled in a 1.5 -mL tube (Eppendorf, Fremont, CA), containing $0.5 \mathrm{~mL}$ of $\mathrm{pH} 7.4$ phosphate-buffered saline and $0.5 \mathrm{~mL}$ of $10 \%$ Triton X-100. The tubes were sonicated for 30 minutes, vortexed for 1 hour, and centrifuged for 30 minutes at $10,000 \mathrm{~g}$ to extract LMWH from the tissues. The amounts of LMWH extracted from the tissues and aqueous were determined with a modified colorimetric method (Azure II; Sigma Aldrich). ${ }^{12}$

\section{Statistical Analysis}

All continuous variables were expressed as the mean $\pm \mathrm{SD}$. The differences in the amount of size decreases of subconjunctival hemorrhages between groups were tested with repeated-measures ANOVA. The baseline sizes and mean elapsed times for the complete resorption of subconjunctival hemorrhages were compared with one-way ANOVA. Post hoc pair-wise comparisons adjusted by the BonferroniDunn method were performed among four groups. As we regarded two eyes of each rabbit as independent subjects, cluster effects was not taken into consideration for statistical analysis. Systemic and ocular absorptions of LMWH were compared by Mann-Whitney test. The level of significance was considered to be $P<0.05$.

\section{Results}

\section{In Vitro Release Rate of LMWH from Liposomes}

The cumulative release rate of LMWH from liposomes is presented in Figure 2. The release of LMWH from liposomes was sustained throughout the study.

\section{Size Decrease of Subconjunctival Hemorrhages}

The size decreases of the subconjunctival hemorrhages are presented in Table 1 . The baseline sizes $( \pm S D)$ of subconjunctival hemorrhages (measured at 8 hours after the hemorrhage induction) were $77.98 \pm 6.26,77.18 \pm 8.66,79.95 \pm 6.35$, and $77.61 \pm 7.49 \mathrm{~mm}^{2}$ in groups $\mathrm{A}, \mathrm{B}, \mathrm{C}$, and $\mathrm{D}$, respectively, which showed no significant differences among all groups $(P=0.750)$. The size decreases in the four groups along all points studied were significantly different $(\mathrm{F}=6.21, P<$ 0.001). Post hoc pair-wise multiple comparisons adjusted by the Bonferroni-Dunn method are presented in Table 2. The size decreases of the subconjunctival hemorrhages were not similar among the four groups at 48, 72, 96, and 120 hours, but were

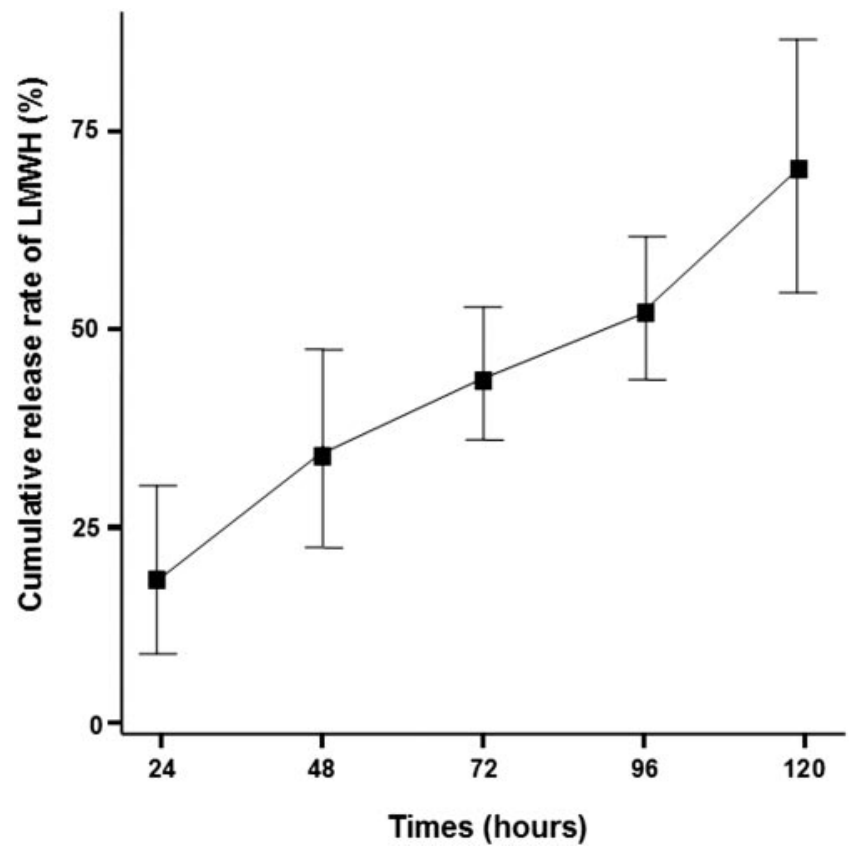

Figure 2. The cumulative rate of in vitro release of LMWH from liposome formulation at 24, 48, 72, 96, and 120 hours after its preparation. Data are expressed as the mean $\pm \mathrm{SD}$. The results suggests that LMWH sustained its release from the liposomes throughout the study $(n=5)$.

similar at 24 hours. The size decreases of subconjunctival hemorrhages occurred significantly faster in group A (liposome-bound LMWH) than in group B (liposomes only) throughout the study, except at 24 hours after the induction $(P=$ $0.617,0.042,0.001,<0.0001$, and $<0.0001$ for $24,48,72,96$, and 120 hours, respectively; Table 2). Comparison of the decreases in size of hemorrhage in groups A and C (free LMWH) showed that they were significantly larger in group A (liposome-bound LMWH) at 96 and 120 hours $(P=0.011$ and 0.001 for 96 and 120 hours, respectively), whereas there were no differences at 24,48 , and 72 hours $(P=0.976,0.592$, and 0.074 for 24,48 , and 72 hours, respectively; Table 2 ).

There were no significant differences in size decreases of subconjunctival hemorrhages between groups $\mathrm{B}$ and $\mathrm{D}$ (no injection) throughout the study $(P=0.950,0.614,0.325$, 0.715 , and 0.744 at $24,48,72,96$, and 120 hours, respectively; Table 2).

\section{Elapsed Time for Complete Resorption of Subconjunctival Hemorrhages}

The mean elapsed times $( \pm \mathrm{SD})$ for the complete resorption of subconjunctival hemorrhage were $134.44 \pm 10.16,184.29 \pm$ $30.72,163.71 \pm 19.18$, and $188.57 \pm 21.27$ hours for group A, $\mathrm{B}, \mathrm{C}$, and D, respectively, and were not similar among the four groups $(\mathrm{F}=22.525, P<0.001)$. Post hoc pair-wise comparisons adjusted by the Bonferroni-Dunn method revealed that the mean time for group A was the shortest among the four groups, whereas there was no significant difference between groups B and D (Fig. 3).

The mean elapsed time for group A was the shortest among the four groups. Group C was next. Mean times for complete resorption of subconjunctival hemorrhages of groups B and D were similar and delayed the most. 
TABLE 1. Size Decreases of Subconjunctival Hemorrhages

\begin{tabular}{ccccc}
\hline $\begin{array}{c}\text { Time } \\
\text { (h) }\end{array}$ & Group A & Group B & Group C & Group D \\
\hline 24 & $7.19 \pm 4.84$ & $5.19 \pm 3.95$ & $7.24 \pm 3.89$ & $5.01 \pm 3.48$ \\
48 & $18.98 \pm 5.25$ & $12.41 \pm 7.67$ & $20.97 \pm 6.88$ & $10.44 \pm 4.65$ \\
72 & $34.50 \pm 10.73$ & $22.24 \pm 14.69$ & $27.58 \pm 6.08$ & $18.32 \pm 8.84$ \\
96 & $47.19 \pm 12.99$ & $29.95 \pm 21.66$ & $37.28 \pm 10.25$ & $28.54 \pm 10.12$ \\
120 & $59.39 \pm 10.27$ & $37.23 \pm 22.02$ & $46.69 \pm 10.47$ & $35.97 \pm 10.03$ \\
\hline
\end{tabular}

Data are expressed as the mean $\left(\mathrm{mm}^{2}\right) \pm \mathrm{SD}$.

\section{Ocular and Systemic Absorption and Intraocular Side Effects of LMWH}

The LMWH concentrations in the aqueous humor and vitreous of eyes treated with free LMWH were significantly higher than those treated with liposome-bound LMWH at 24 and 48 hours $(P=0.028$ at 24 hours and 0.003 at 48 hours for aqueous humor; $P=0.036$ at 24 hours and 0.001 at 48 hours for vitreous). Then, the activities of LMWH in the vitreous of eyes treated with free LMWH decreased to the level of no significant differences compared with those treated with liposome-bound LMWH at 72 and 120 hours $(P=0.375$ at 72 hours and 0.428 at 120 hours for aqueous humor; $P=0.129$ at 72 hours and 0.289 at 120 hours for vitreous; Figs. $4 \mathrm{~A}, 4 \mathrm{~B})$.

The LMWH activities were significantly higher in the conjunctiva and sclera at 24 and 48 hours $(P=0.017$ and 0.008 , respectively, for conjunctiva; $P=0.034$ and 0.023 , respectively for sclera) after free LMWH injection compared with those after liposome-bound LMWH (Figs. 4C, 4D). Then, the activity in the conjunctiva and sclera were significantly lower in eyes treated with free LMWH, whereas sustained activity was shown with liposome-bound LMWH at 72 and 120 hours ( $P=0.017$ at 72 hours and 0.009 at 120 hours for conjunctiva; $P=0.021$ at 72 hours and 0.011 at 120 hours for sclera).

Systemic absorption after treatment with liposome-bound LMWH was significantly less at 24 and 48 hours compared with that after free form of LMWH $(P=0.37$ and 0.45 , respectively; Fig. 4E).

There were no detectable LMWH in the fellow untreated eyes (aqueous, vitreous, conjunctiva, and sclera) after subconjunctival injection of liposome-bound or free LMWH throughout the study.
Slit lamp and fundus examinations revealed no evidences of opacities in the cornea or lens and inflammations or hemorrhages in the anterior chamber and vitreous before and at 24, 48, 72, and 120 hours after subconjunctival hemorrhage induction with subsequent injection of liposome-bound or free form of LMWH.

\section{Discussion}

The results of our study suggest that subconjunctival injection of liposome-bound LMWH enhances the rate of subconjunctival hemorrhage resorption steadily with less intraocular or systemic absorption, although the free form of LMWH retained this effect in the relatively early phases, but not in the later phases. The mechanisms by which LMWH facilitated the resorption of subconjunctival hemorrhages might be explained as follows. First, thrombin-activatable fibrinolysis inhibitor (TAFI) is the precursor of an exopeptidase that is identical with plasma procarboxypeptidase $\mathrm{B}^{7}$ On activation by thrombin, activated TAFI attenuates fibrinolysis, presumably by catalyzing the removal of C-terminal lysines from partially degraded fibrin. Thus, as inhibitors of TAFI activation may enhance thrombolysis, ${ }^{13}$ anti-thrombin III activity of LMWH would be expected to enhance the resorption of subconjunctival hemorrhage. Second, LMWH-induced lipase activity may also enhance subconjunctival hemorrhage resorption. ${ }^{14}$ Because the plasma membrane of red blood cell is composed of a lipid bilayer, any local increase in lipase activity would accelerate red blood cell degradation. Third, LMWH is known to sequester fibroblast growth factor 2 (FGF2) in the extracellular environment, which upregulates urokinase-type plasminogen activator (uPA)

Table 2. Post Hoc Pair-Wise Multiple Comparison of the Size Decrease of Subconjunctival Hemorrhages

\begin{tabular}{|c|c|c|c|c|c|c|}
\hline \multirow{2}{*}{\multicolumn{2}{|c|}{$\begin{array}{c}\text { Multiple } \\
\text { Comparisons } \\
\text { among Groups }\end{array}$}} & \multicolumn{5}{|c|}{ Time (h) } \\
\hline & & 24 & 48 & 72 & 96 & 120 \\
\hline \multirow[t]{3}{*}{ A } & B & 0.617 & $0.042^{*}$ & $0.001^{*}$ & $<0.0001^{*}$ & $<0.0001^{*}$ \\
\hline & $\mathrm{C}$ & 0.976 & 0.592 & 0.074 & $0.011^{*}$ & $0.001^{*}$ \\
\hline & $\mathrm{D}$ & 0.570 & $0.026^{*}$ & $<0.0001^{*}$ & $<0.0001^{*}$ & $<0.0001^{*}$ \\
\hline \multirow[t]{2}{*}{ B } & $\mathrm{C}$ & 0.542 & $0.036^{*}$ & 0.192 & 0.272 & 0.151 \\
\hline & $\mathrm{D}$ & 0.950 & 0.614 & 0.325 & 0.715 & 0.744 \\
\hline C & $\mathrm{D}$ & 0.574 & $0.009^{*}$ & $0.022^{*}$ & 0.131 & 0.238 \\
\hline
\end{tabular}

Data are probabilities obtained with repeated-measures ANOVA. The subconjunctival hemorrhages decreased in size significantly faster in group A (liposome-bound LMWH) than in group B (liposomes only) throughout the study, except at 24 hours after induction. Comparison of groups A and C (free LMWH) showed were significantly larger decreases in group A (liposome-bound LMWH) at 96 and 120 hours, with no differences at 24,48 , and 72 hours.

$$
\text { * } P<0.05 \text {. }
$$




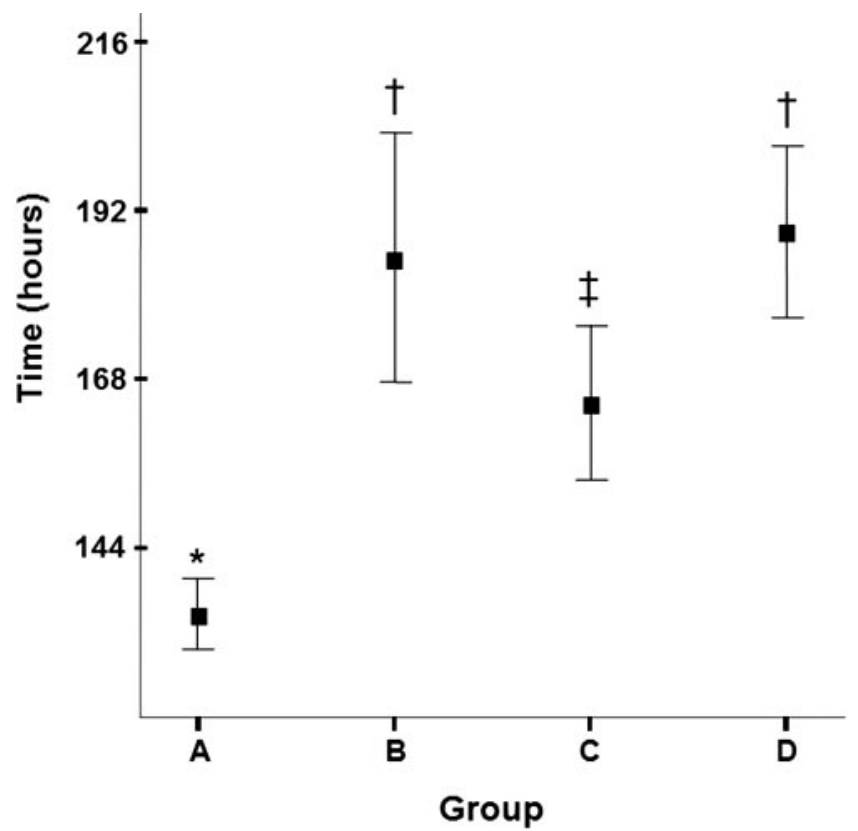

Figure 3. Post hoc groupings after comparison of the mean elapsed times for the complete resorption of subconjunctival hemorrhages among the four study groups. The mean elapsed time for group A was shortest among the four groups, followed by group $\mathrm{C}$, and, then groups $\mathrm{B}$ and $\mathrm{D}$. Data are expressed as the mean $\pm \mathrm{SD}$. ${ }^{*}+\neq$ The group(s) is(are) different from the rest.

and matrix metalloproteinase (MMP) production in vascular endothelial cells. ${ }^{15}$ Thus, blood resorption may be enhanced because uPA converts plasminogen into plasmin and activates several MMPs which participate in extracellular matrix degradation. ${ }^{6,16}$ Fourth, endothelial modulation by LMWH has been reported to induce the release of fibrinolytic activators such as tissue plasminogen activator and antiplatelet substances like prostacyclin. ${ }^{5}$ Finally, clot retraction, during which the contractile force is transmitted via platelet glycoprotein IIb/IIIa receptors that bind the polymerized fibrin network to the actin cytoskeletons of platelets, is known to make blood clots more resistant to fibrinolysis. ${ }^{17}$ In view of these relations, it might be speculated that the suppression of fibrin meshwork formation by LMWH makes blood clots more susceptible to fibrinolysis.

As shown in Table 2, the thrombolysis-enhancing effect of free LMWH held on only in the early phases of this study (at 24, 48, and 72 hours after the induction of hemorrhage), however, no longer in the later phases (at 96 and 120 hours) compared with that of liposome-bound LMWH. In a perspective of doseresponse relationship, these findings correspond well with the results that the LMWH activity in conjunctival and sclera adjacent to the injection site was significantly higher at 24 and 48 hours after hemorrhage induction with a subsequent decline in activity, whereas liposome-bound LMWH maintained its local activity through sustained release from liposomes throughout the study (Figs. 4C, 4D). Local retention of liposome-bound LMWH and its sustained release from liposomes are most likely to be the causes of maintaining local LMWH activity in the conjunctival and sclera.

Ideal ocular LMWH delivery systems for the purpose of this study should not only enhance the focal retention and sustained release, but should also prevent systemic side effects and the ocular toxicity of LMWH. Moreover, the sustained release and local retention of $\mathrm{LMWH}$ is imperative due to a narrow therapeutic index of LMWH $(0.6-1.0 \mathrm{IU} / \mathrm{mL})$ to overcome the risks for high initial LMWH concentrations at the site of injection and the drainage of LMWH to systemic circulation,
A

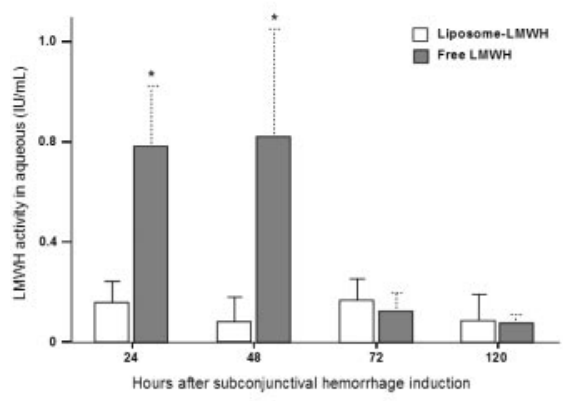

c

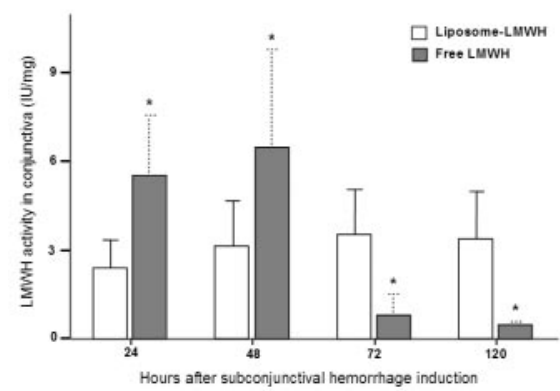

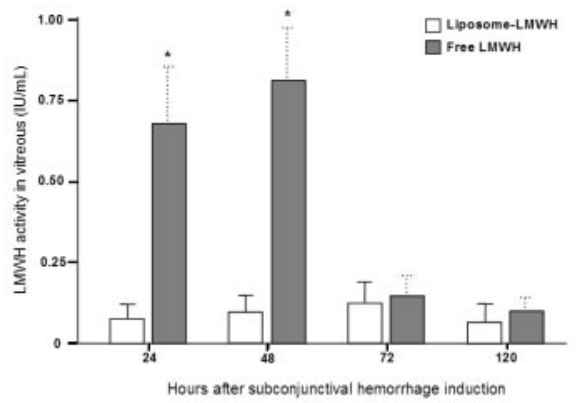

D

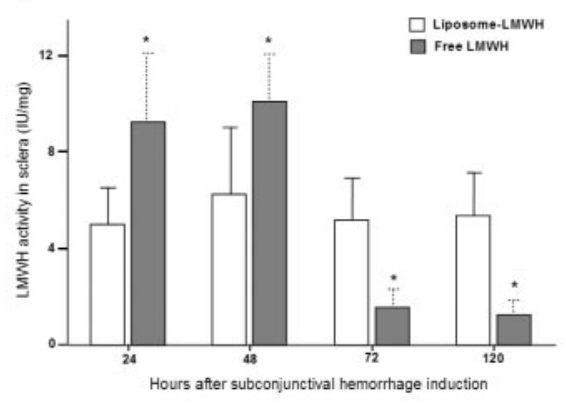

E

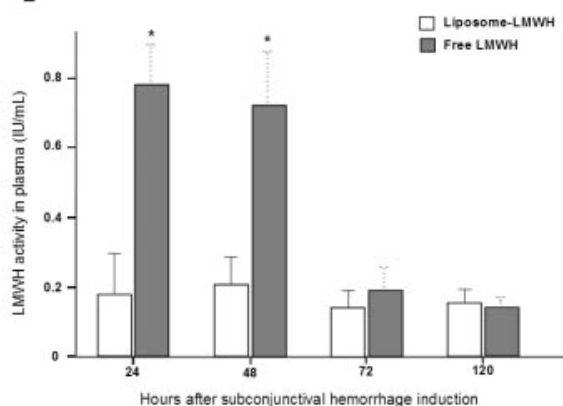

Figure 4. Comparison of the concentrations of LMWH in aqueous humor (A), vitreous (B), conjunctiva (C), sclera (D), and plasma (E) between eyes treated with liposome-bound LMWH and eyes with the free form of LMWH at 24, 48, 72, and 120 hours after subconjunctival hemorrhage induction. Data are expressed as the mean $\pm \mathrm{SD} .{ }^{*} P<0.05$. 
which may cause side effects such as bleeding, thrombocytopenia, hypersensitivity reactions, and osteoporosis. ${ }^{18}$ Liposomes work well in this context, as intraocular (aqueous and vitreous) and systemic absorption of LMWH was considerably less in group A (liposome-bound LMWH) than group $\mathrm{C}$ (free LMWH) in our study (Figs. 4A, 4B, 4E). This may be explained as follows. First, the large size of the liposomes ensures that they remain localized at the injection site. ${ }^{19}$ This explains why there was significantly lower LMWH activity in the aqueous and vitreous of eyes treated with liposome-bound LMWH (group A) compared with the free form of LMWH (group C) in our study (Fig. 4A, 4B). Although intraocular side effects were not detectable in either group, lower intraocular absorption may be desirable because of the potential risk of side effects. Ambati et al. ${ }^{20}$ demonstrated that scleral permeability declines exponentially with increasing molecular radius (from 0.5 to $8.25 \mathrm{~nm}$ ) and that molecular radius is a better predictor of scleral permeability than is molecular weight. It may be possible that because of the large molecular radius of the liposomes used in our study ( $546 \pm 41.94 \mathrm{~nm}$ ), it could not be introduced into intraocular region, thus resulting in intrascleral entrapment as presented in Figure 4D. In addition, the molecules, with a molecular mass of 4 to $150,000 \mathrm{D}$, were found to diffuse across the sclera in a manner consistent with porous diffusion through a fiber matrix. ${ }^{20}$ Consequently, high LMWH activity in the aqueous and vitreous of eyes treated with free LMWH injection, with a mass of $\sim 7000 \mathrm{D}$ in our study, should be attributed to the feasibility of transscleral penetration of free LMWH.

Second, liposomes may block lymphatic and other drainage pathways, and thereby retard the rate of free drug clearance. $^{21,22}$ This, along with sustained release of LMWH from liposomes, ${ }^{8,9}$ explains in part why there was less systemic LMWH activity after liposome injection (Fig. 4E).

Third, the positively charged liposomes used in this study enable loading of large doses of drug, and increase the drug residence time and concentration for adsorption at the target sites, according to studies by Law and Hung. ${ }^{10}$ In the previous work, the stability and the entrapment efficiency of LMWHcontaining liposomes were greatly influenced by the liposomal surface charge. The positively charged liposomes showed higher stability and entrapment efficiency than the neutral or negatively charged ones. ${ }^{23}$

Although topical application of liposomes was feasible in this study, subconjunctival injection of liposomes was reported to provide effective therapy through directly targeting the site of action and facilitate the slow, steady release of the encapsulated drug, thus allowing a high drug concentration at the desired site for a long-lasting time. ${ }^{8}$ Moreover, the main benefit of a subconjunctival route would be the improved compliance of patients, because topical preparations can require frequent administration.

LMWH has some significant advantages over unfractionated heparins for the purpose of this study. First, a longer half-life by two- to fourfold. ${ }^{2}$ Compared with the short (approximately 1.5 hours) and variable half-life of unfractionated heparin, ${ }^{24}$ LMWH has a longer and more consistent half-life. ${ }^{24,25}$ The disappearance times of antifactor $\mathrm{Xa}$ and antithrombin activity of LMWHs have been reported to be longer than 16 hours. ${ }^{26}$ These findings may explain in part the sustained effect of liposome-bound LMWH. Second, LMWH administration is associated with a lower bleeding propensity, as the anti-Xa to anti-thrombin inhibition ratios of unfractionated heparin and LMWH are $1: 1$ and 2 to $4: 1$, respectively. ${ }^{2,25}$ The less tendency of LMWH to induce hemorrhage is another reason that we selected LMWH instead of unfractionated heparin in this study.
There are a few limitations in this study. First, as we did not consider the curvature of the rabbit eyes, the size measurement through picturing the lesions on the ocular surface may not reflect the actual size. To minimize this error, we tried to picture right above the center of the hemorrhagic lesion at a fixed distance and magnification with a reference ruler. Second, the subconjunctival concentration of LMWH was not checked. The lack of this data may prevent the direct association between the subconjunctival application of LMWH and its effect at the site. The absorption amount of LMWH after subconjunctival injection in the conjunctiva and sclera may reflect its subconjunctival concentration. Third, as the subconjunctival hemorrhages in our model were contrived, the action of liposome-bound LMWH on spontaneous cases remains undetermined.

In conclusion, our findings suggest that the subconjunctival injection of liposome-bound LMWH enhances the absorption of subconjunctival hemorrhage in rabbit eyes, and could be useful for treating subconjunctival hemorrhage.

\section{Acknowledgments}

The authors thank Jong-Ju Lee, MD, for meticulous technical support and the Medical Research Collaborating Center, Seoul National University College of Medicine, Seoul National University Hospital, for statistical advice.

\section{References}

1. Richards RD. Subconjunctival hemorrhage: treatment with air therapy. Eye Ear Nose Throat Monogr. 1965;44:59.

2. Kleinschmidt K, Charles R. Pharmacology of low molecular weight heparins. Emerg Med Clin North Am. 2001;19:1025-1049.

3. Klein W, Buchwald A, Hillis SE, et al. Comparison of low-molecular-weight heparin with unfractionated heparin acutely and with placebo for 6 weeks in the management of unstable coronary artery disease. Circulation. 1997;96:61-68.

4. Simonneau G, Sors H, Charbonnier B, et al. A comparison of low-molecular-weight heparin with unfractionated heparin for acute pulmonary embolism. N Engl J Med. 1997;337:663-669.

5. Fareed J, Jeske W, Hoppensteadt D, Clarizio R, Walenga JM. Lowmolecular-weight heparins: pharmacologic profile and product differentiation. Am J Cardiol. 1998;82:3L-10L.

6. Hiraoka N, Allen E, Apel IJ, Gyetko MR, Weiss SJ. Matrix metalloproteinases regulate neovascularization by acting as pericellular fibrinolysins. Cell. 1998;95:365-377.

7. Nesheim M, Wang W, Boffa M, Nagashima M, Morser J, Bajzar L. Thrombin, thrombomodulin and TAFI in the molecular link between coagulation and fibrinolysis. Thromb Haemost. 1997;78: 386-391.

8. Assil KK, Frucht-Perry J, Ziegler E, Schanzlin DJ, Schneiderman T, Weinreb RN. Tobramycin liposomes: single subconjunctival therapy of pseudomonal keratitis. Invest Ophthalmol Vis. Sci. 1991; 32:3216-3220.

9. Barza M, Baum J, Szoka F Jr. Pharmacokinetics of subconjunctival liposome-encapsulated gentamicin in normal rabbit eyes. Invest Ophthalmol Vis. Sci. 1984;25:486-490.

10. Law SL, Hung HY. Properties of acyclovir-containing liposomes for potential ocular delivery. Int J Pharm. 1998;161:253-259.

11. Touitou E, Dayan N, Bergelson L, Godin B, Eliaz M. Ethosomes: novel vesicular carriers for enhanced delivery: characterization and skin penetration properties. J Control Rel. 2000;65:403-418.

12. Jiao Y, Ubrich N, Hoffart V, et al. Anticoagulant activity of heparin following oral administration of heparin-loaded microparticles in rabbits. J Pharm Sci. 2002;91:760-768.

13. Bajzar L, Nesheim ME, Tracy PB. The profibrinolytic effect of activated protein Cin clots formed from plasma is TAFI-dependent. Blood. 1996;88:2093-2100.

14. Perry WF. Heparin-activated lipase in diabetic, atherosclerotic and healthy subjects. Clin Chim Acta. 1967;16:189-194. 
15. Presta M, Dell'era P, Mitola S, Moroni E, Ronca R, Rusnati M Fibroblast growth factor/fibroblast growth factor receptor system in angiogenesis. Cytokine Growth Factor Rev. 2005;16:159-178.

16. Liotta LA, Steeg PS, Stetler-Stevenson WG. Cancer metastasis and angiogenesis: an imbalance of positive and negative regulation. Cell. 1991;64:327-336.

17. Kunitada S, FitzGerald GA, Fitzgerald DJ. Inhibition of clot lysis and decreased binding of tissue-type plasminogen activator as a consequence of clot retraction. Blood. 1992;79:1420-1427.

18. Hirsh J, Anand SS, Halperin JL, Fuster V. Mechanism of action and pharmacology of unfractionated heparin. Arterioscler Thromb Vasc Biol. 2001;21:1094-1096.

19. Assil KK, Hartzer M, Weinreb RN, Nehorayan M, Ward T, Blumenkranz M. Liposome suppression of proliferative vitreoretinopathy. Invest Ophthalmol Vis. Sci. 1991;32:2891-2897.

20. Ambati J, Gragoudas ES, Miller JW, et al. Transscleral delivery of bioactive protein to the choroid and retina. Invest Opbthalmol Vis Sci. 2000;41:1186-1191.
21. Barza M, Stuart M, Szoka F Jr. Effect of size and lipid composition on the pharmacokinetics of intravitreal liposomes. Invest Opbthalmol Vis Sci. 1987;28:893-900.

22. Fitzgerald P, Hadgraft J, Wilson CG. A gamma scintigraphic evaluation of the precorneal residence of liposomal formulations in the rabbit. J Pbarm Pharmacol. 1987;39:487-490.

23. Song YK, Kim CK. Topical delivery of low-molecular-weight heparin with surface-charged flexible liposomes. Biomaterials. 2006; 27:271-280.

24. Hirsh J, Warkentin TE, Raschke R, Granger C, Ohman EM, Dalen JE. Heparin and low-molecular-weight heparin: mechanisms of action, pharmacokinetics, dosing considerations, monitoring, efficacy, and safety. Chest. 1998;114:489S-510S.

25. Estes JW. Clinical pharmacokinetics of heparin. Clin Pharmacokinet. 1980;5:204-220.

26. Heit J. Low-molecular-weight heparin: biochemistry, pharmacology, and concurrent drug precautions. Reg Anesth Pain Med. 1998;23:135-139. 